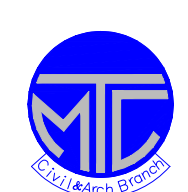

ICCAE

Military Technical College

Kobry Elkobbah,

Cairo, Egypt

\author{
7th International Conference \\ On Civil \& Architecture \\ Engineering
}

\title{
ASSESSMENT OF TOPOGRAPHIC MAPS PRODUCED BY DIGITAL PHOTOGRAMMETRIC WORKSTATION
}

MOUSSA $^{*}$ O.M., RAGAB ${ }^{* *}$ A.F. and KHEDRE ${ }^{* * *}$ N.A.

\begin{abstract}
Topogeaphic map can be considered as an essential initial product for most of large engineering projects. Hence, increasing the efficiency and quality of these maps is a main issue of any surveying works. Many methods are considered in the production of topographic maps, according to the required accuracy, available instruments, afforded time, and involved personnel. The history of topographic map production by photogrammetry is not very old, only about half a century, due to its great efficiency and advantage especially in inaccessible and mountaneous areas. Today, photogrammetry has been vastly broadened to include the use of data from sensors utilizing other portions of the electromagnetic spectrum, in additional to the conventional photography, for many applications. Also, as a result of the rapid development of computer technology and software, assessment of the accuracy of the topographic map produced by applying the principle of digital photogrammetric workstation is the main scope of the current investigation.

In order to achieve this objective, aerial panchromatic photos have been taken at a scale of 1:15000 for a certain hilly study area, and scanned at different resolutions. Leica photogrammetry suite (LPS) digital software was used to perform this work with reasonable accuracy. The final resulted values as discrepancies of some selected check points, root mean square errors, total image unit-weight RMSE, and standard deviations of the automatic generated tie poits shows that 2117 dpi resolution, which
\end{abstract}


equivalent to an image coverage $12 \mu \mathrm{m}$, gives the less error values than the other adopted resolutions and with an absolute optimistic accuracy. In addition, the topographic map, presented as a layer of spot points, resulted from this resolution will be compared with the available base topographic map for the same study area produced from analytical stereoplotters device, by using special map projection software for unifying the coordinates of both analytical and digital map. From this comparison, it can be found that, about $75 \%$ percent of the resulted points passed on contour's reference topographic map, which indicate the efficieny of the topographic maps produced by digital photogrammetric workstations.

\footnotetext{
*Professor, Chair of Civil Engineering, Military Technical College, Cairo, Egypt.

** Associate professor, Dpt. of Public Works, Ain Shams University, Cairo, Egypt.

${ }^{* * *}$ Colonel, Syrian Defense.
}

\section{INTRODUCTION}

Advanced technologies in all sciences, concerning topographic map production, are developed in methods and processing. Previously, topographic map production was dependant on the field works, which require numerous civil engineers in order to achieve the job, different instruments, repeating of measurements, adjustments, availability of time, and high cost. Then, analytical stereoplotters devices were used to produce topographic maps. This, in turn, needs persons that have high experience and good vision to work on these devices. Nowadays, topographic maps produced from digital photogrammetry workstations depend on software, and most operations are automatically extracted. So, digital photogrammetry has matured and superseded the analytical photogrammetry in terms of accuracy, production, efficiency and multiple usage [2]. Accordingly, most countries in the world use the digital photogrammetry workstations, in order to produce topographic maps.

In this context, digital technology has also penetrated in all aspects of the mapping process, from raw data collection through map compilation and design to final production. Also, Advancement in mapping technology has replaced the conventional photogrammetric analytical stereoplotters with digital imaging systems, and then digital photogrammetric workstation. Consequently, the technological advancements has made map making more efficient and accurate [1], since the digital map is stored in real world coordinates and can be plotted at any scale required [3]. Moreover, this rapid technological development has been also involved with the cartography which is the conception, production, dissemination, and study of maps in all forms. So, digital photogrammetry is a promising approach for producing topographic maps.

Accordingly, the main objective of this research is to produce a topographic map showing contour lines with reasonable accuracy by using Leica photogrammetry suite software (LPS) as the used digital photogrammetric workstation. Of course, the reasonable accuracy depends on the corresponding scanned resolution, and hence, the overall operational cost, including the used scanner, time, file size, and the specification of the used hardware for data analysis. The resulted contour lines from 
different resolutions are analyzed on the basis of discrepancies of the selected check point's coordinates, root mean square errors, and the least squares adjustment information presented in the associated aerial triangulation reports. Moreover, The produced contour lines from the best resolution will be compared with the available base (reference) map, produced from analytical stereoplotters and contains the same study area. Accordingly, the percentage of points which passed on the reference map related to their heights is also taken into account in the final assessment of the digitally produced topographic map.

\section{DATA ACQUISITION}

The used data for the selected study area include six black-white photos, which are scanned with high-precision scanner at $1016 \mathrm{dpi}, 1588 \mathrm{dpi}$, and $2117 \mathrm{dpi}$ resolutions, respectively, and stored in workstation's hardware, since the scanning resolution is very critical for achieving the accuracy of any product from the used digital photogrammetric workstation. These resolutions are equivalent to 25 microns, 16 microns, and 12 microns, respectively. This study area is located near Cairo city and bounded between $30^{\circ} 01^{\prime} 15.22 " \mathrm{~N}, 30^{\circ} 04^{\prime} 14.37^{\prime \prime} \mathrm{N}$, and between $31^{\circ} 23^{\prime} 25.16^{\prime \prime} \mathrm{E}$, $31^{\circ} 25^{\prime} 47.87^{\prime \prime} \mathrm{E}$, as shown in Fig. 1 . These photos have been taken by camera type IGN1-1043, whose calibrated focal length is $151.79 \mathrm{~mm}$, and with an approximate average scale 1:15000. These information are listed in the Table 1.

In addition, some base points are identified in the block, whose 3D Cartesian ground coordinates are known. Some of these points are treated as control points, while the others are treated as check points. After numbers of solution tries, a certain selection and distribution of these base points over the study area is decided to give the optimum number and distribution of both control and check points, in order to give suitable results for aerial triangulation. This distribution is depicted in Fig. 2. It should be noted that, both ground and check points are placed on the rocks and are not very clear, which should be considerd in the final assessment of the produced topographic map.

\section{METHODOLOGY OF INVESTIGATION}

In order to construct a topographic map along with contour lines from aerial photographs, Leica photogrammetry suite software (LPS) version 8.7 was used for this purpose. Nowadays, most surveyors use digital photogrammetric workstation for maps production, because they reduce time, effort, cost, and give a reasonable accuracy.

As stated before, the main objective of the current investigation here, is to obtain the results of triangulation from digital photogrammetric workstation, according to ground control and check points which were chosen, and automatic generated tie points. Some of these tie points having high residuals should be adjusted or even deleted. After accepting the root mean square error RMSE in the triangulation report associated with the used software, digital terrain model DTM are extracted and some points in the resulted DTM are checked to assess the final accuracy.

Three triangulation reports were obtained according to the three used scanning resolutions. One can compute the discrepancies in the 3D ground coordinates $(\mathrm{X}, \mathrm{Y}$, $Z$ ) of all selected check point, which is simply the difference between the computed 
coordinates from aerial triangulation and the actual reference ground coordinates in the three directions. In addition and for instance, the discrepancies of spatial position for any block (according to each used resolution), since it is generally the most important parameter used to estimate the quality of any topographic data [4], will be evaluated in the following manner:

$$
\operatorname{Disc} P=\sqrt{(\operatorname{Disc} X)^{2}+(\operatorname{Disc} Y)^{2}+(\operatorname{Disc} Z)^{2}}
$$

Where:

DiscP: the discrepancy of spatial position for any check point in the block.

Disc $X$, DiscY, DiscZ: the discrepancies in three directions $X, Y, Z$ for the same check point.

Moreover, from the results in triangulation reports, the efficiency of each scanning resolution will be evaluated by analyzing the root mean square error RMSE of the 3D and positional discrepancies at all selected check points for the whole block, since it is the most widely and popular index for error measurements [5]. Also, the standard deviations of the computed ground coordinates of all check and tie points related to each scanning resolution will be studied.

Finally, one of the available data is a hardcopy base topographic map for the same study produced from analytical stereoplotters, with a scale of 1:25000. The map was scanned at $2117 \mathrm{dpi}$ and saved in the environment of the AutoCAD software as a layer. on other hand, the coordinates of all object points resulted from the expected best resolution $2117 \mathrm{dpi}$, that resulted from aerial triangulation process, are transformed from the output grid coordinate system $(E, N)$ into the corresponding projected angular coordinate system $(\varphi, \lambda)$ by using of special software for map projection, in order to match the original base map coordinates. Therefore, the transformed coordinates are processed by the Golden Surfer software, in order to draw them as spot points in a worksheet. This worksheet was overlyed to the topographic map, which is presented in AutoCAD software as layer, in order to study the matching of the position of the resulted spot points with the scanned contour lines of the available base topographic map. From this overlying, one can show if the spot points are located between the two contour lines that accommodate their heights, and compute the number of points which are passed.

\section{CRITERIA OF ASSESSMENT}

As has been stated in the methodology of investigation, in the previous section, and in order to study the effect of using different scanning resolutions on the accuracy of the produced topographic map, besides assessing this output accuracy related to availabe base map, four evaluation criteria have been suggested to test the efficiency of the different resolutions. The first criterion is concerned with the discrepancies in the 3D ground cordinates and position at all selected check points in the block as they have been evaluated, in the three used resolutions, from equation (1). The second criterion is comparing the residuals and accuracies of the resulted exterior orientation parameters. The third criterion is comparing the resulted accuracies of the selected tie points, included in triangulation report related to each resolution. Finally, the fourth criterion is the assessment of the final accuracy by plotting the resulted points from aerial triangulation, using Golden Surfer software, 
and overlying these points to the topographic map resulted from stereoplotters and presented by the AutoCAD software.

\section{PRESENTATION AND ANALYSIS OF THE OBTAINED RESULTS}

In order to analyze the obtained results from aerial triangulation corresponding to each adopted scanning resolution, according to the criteria in the previous section, the following parameters must be taken into account to obtain the best resolution, which gives the reasonable accuracy and its results will be taken to assess the produced topographic map. In the first criterion, the statistical parameters of these discrepancies will be computed, as the mean, maximum, minimum, range, and RMS in three directions $X, Y$, and $Z$, as well as the RMS of the spatial position for the whole block. These values will be given in the Table 2. From this table, it is quite obvious that, the resolution 2117 dpi gave less discrepancies values for all parameters in the spatial position for the tested block, than other resolutions 1588 dpi, and 1016 dpi. For example, the resolution 2117 dpi gives an improvement of about $10 \%$ in the RMS values of the positional discrepancies compared with resolution $1588 \mathrm{dpi}$, and an improvement of about 30\% than resolution $1016 \mathrm{dpi}$. Therefore, it can be regarded that the resolution $2117 \mathrm{dpi}$ as the best resolution.

It should be noted here that, it is an expected result since the main concern of this criterion is not the relative comparison between the different scanning resolutions, but the assessment of each one related to the known reference ground coordinates of some selected check points. For example, the resolution 2117 dpi gives an accuracy of about $1.77 \mathrm{~m}$ relative to the base coordinates, which can be considered an optimistic value, taking into account the scale of the photos and the accuracy of the base ground coordinates. In addition, and according to the same purpose, this criterion indicates also to which maximum resolution can be adopted to give the required final accuracy, of course, without increasing the cost and file size, since there is no large gap between the two scanning resolutions $2117 \mathrm{dpi}$ and $1588 \mathrm{dpi}$ on all computed statistical parameters of the resulted discrepancies.

The second criterion includes the output results of the exterior orientation parameters (three rotation angles " $\omega, \varphi, k$ " and the three ground coordinates of each exposure station " $\mathrm{X}_{\mathrm{L}}, \mathrm{Y}_{\mathrm{L}}, \mathrm{Z}_{\mathrm{L}}$ ") of each photo in the whole block corresponding to each adopted scanning resolution. In other words, it investgates the output residuals of these parameters, as depicted in Table 3, and the corresponding output accuracies (expressed as standard deviations), related to assumed initial values of standard deviations for the exposure station ground coordinates and the three rotation angles as $1.0 \mathrm{~m}$, and 0.1 degree, respectively. These results are also listed in Table 3.

From the results shown in Table 3, the residuals of the exterior orientation parameters of the block corresponding to resolution $2117 \mathrm{dpi}$, in the three coordinate directions and the three rotation angles, are less in values than the other residuals, which resulted from resolutions $1588 \mathrm{dpi}$ and $1016 \mathrm{dpi}$. This indicates that the resolution $2117 \mathrm{dpi}$ is the best. Also, Table 3 illustrates that the accuracies of the exterior orientation parameters are increased with increasing the resolution. In other word, the accuracies of the exterior orientation parameters of the block is more improved at the resolution $2117 \mathrm{dpi}$. Then, this resolution can be again regarded as the best resolution to be adopted. It should be noted that, the improvement in both 
residuals and accuracies of the exteior orientation parameters are satisfied in each image, but the values related to each parameter in Table 3 are listed as the average values only for the six images forming the block.

The third criterion of accuracy assessment depends on the triangulation results of the selected tie points, described by their standard deviations and root mean square errors for each adopted scanning resolution, as listed in Table 4. From that table, one can clearly find that, the resultant total RMSE, mean, and standard deviations of the tie points of the whole block increase with the decreasing resolution, which verify the findings from the previous two criteria.

Finally, the fourth criterion includes the comparison between the ground points coordinates resulted from using the best resolution with available topographic map produced from analytical stereoplotters. Fig. 3 shows the comparison between ground points coordinates produced by using the best resolution $2117 \mathrm{dpi}$, plotted as spots, and overlying these spots (as layer) to the topographic map, resulted from analytical stereoplotters and presented by AutoCAD software, at different parts of the study area. From this comparison, it was found that, the percentage of the spots which passed on the available digitized contour lines of the reference topographic map equals about $75 \%$. This percentage gives a sufficient accuracy to production of topographic map totally by using the digital photogrammetric workstations. In addition and as stated before, this obtained accuracy can be improved by increasing the accuracy of the used ground control points, which it is not the case here since most of the used ground control points are not clearly identified.

\section{CONCLUSIONS}

The main conclusions can be extracted and summarized in the following enumeration:

1- Distribution of ground control points plays the important role on the final accuracy of the resulted topographic maps.

2- The least 3D discrepancies in check points coordinates resulted from scanned aerial photographs with high resolution through the digital photogrammetric workstation.

3- The selected scanning resolution should be matched with the required final accuracy.

4- Increasing the scanning resolution helps, with a considerable level, in increasing the number of tie points that are measured automatically.

5- In most cases, the output digital relief should be subjected to what is socalled map projection, in order to be comparable with the available printed hardcopy commercial topographic maps.

6- The resulted aerial triangulation points from LPS digital photogrammetric software were representing an accurate relief, when compared with the topographic map produced from analytical stereoplotters.

As an overall conclusion, it is worthwhile to mention that, the digital photogrammetric workstations can be considered as efficient and optimum tools for producing topographic maps, even for hilly and mountaneous area. For achieving that, accuracy and distribution of ground control points, and the suitable selected scanning resolution should be carefully considred. 


\section{REFERENCES}

[1] Bolastad, P., "A first Text on Geographic Information Systems", (2005).

[2] Chaturvedi, A.; W. Bell; K. Rochester and B. Acharya, "Optimizing the Presentday Photogrammetric Operations", IAPRS, Vol. XXXIII, Amsterdam, (2000).

[3] Kheder, N. A., " Studying and Designing of Topographic Maps (Cartography)", M.Sc. Thesis, Military Technical College, Chair of Civil Engineering, Egypt, (2007).

[4] Mao, H. X.; W. Z. Shi and Y. Tian, "A preliminary Study on Spatial Sampling for Topographic Data", (2005).

[5] Weng, Q., " Quantifying Uncertainity of Digital Elevation Models Derived from Topographic Maps", (2001).

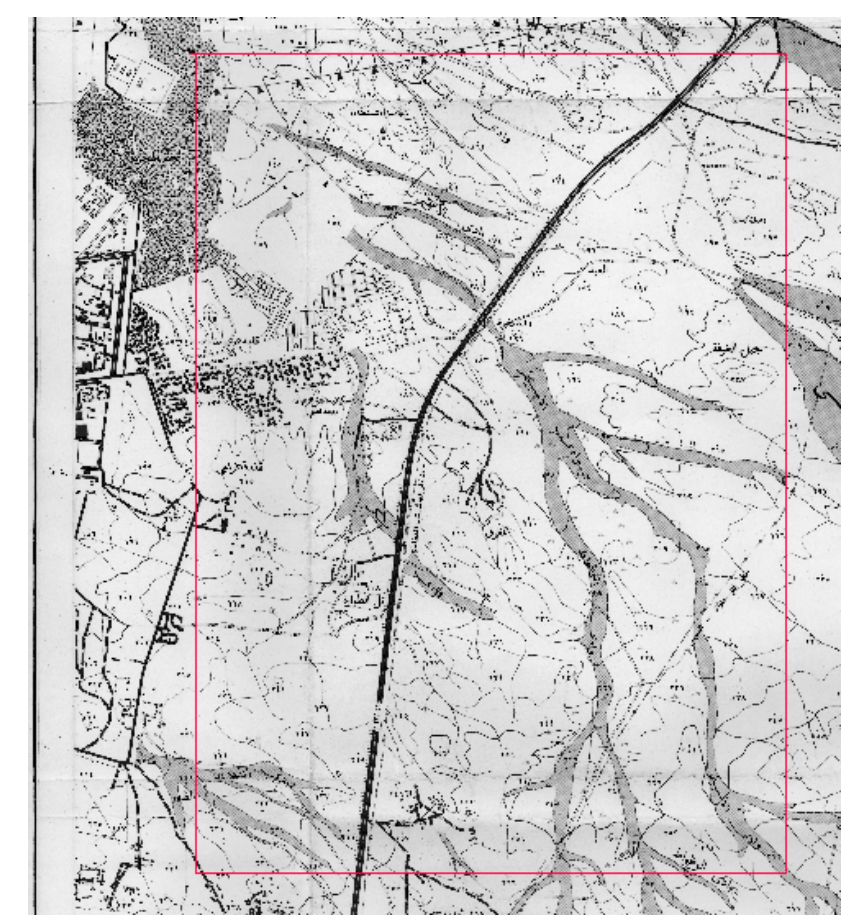

Fig. 1. Tested Area 


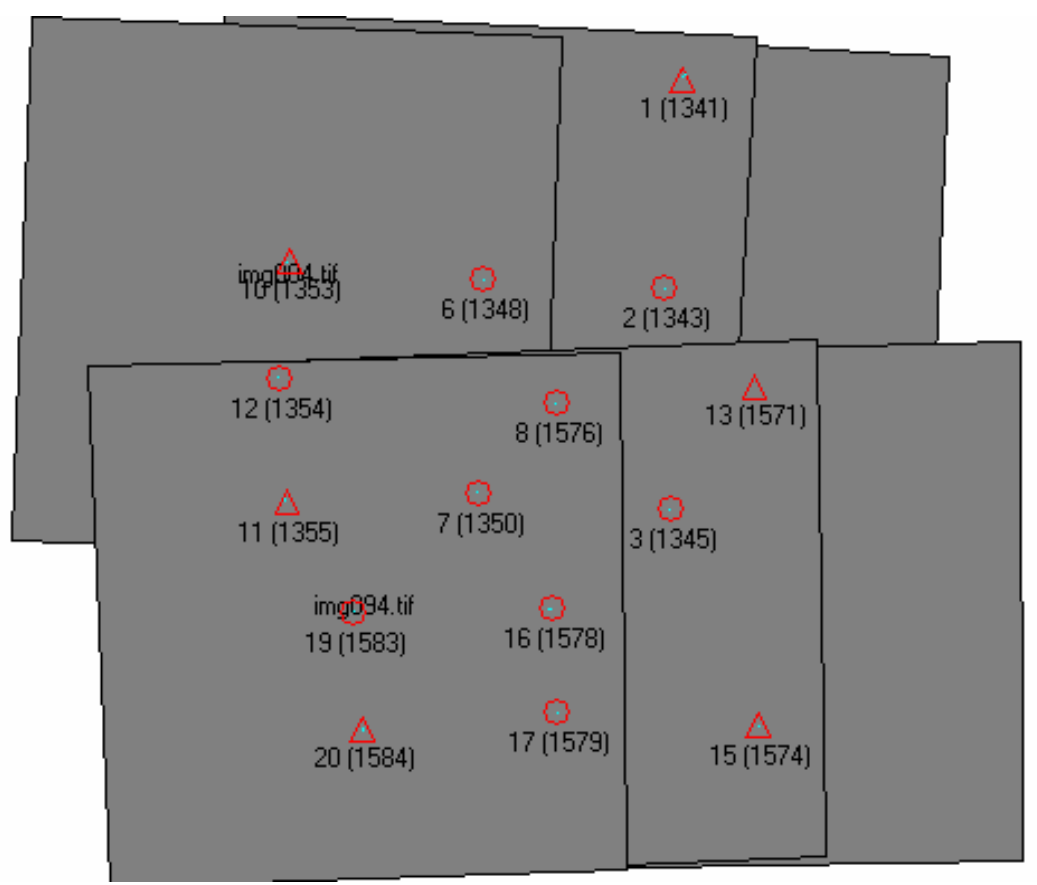

Fig. 2. Distribution of Ground Control and Check Points in the Block $\Delta$ Control Point, $\circ$ Check Point
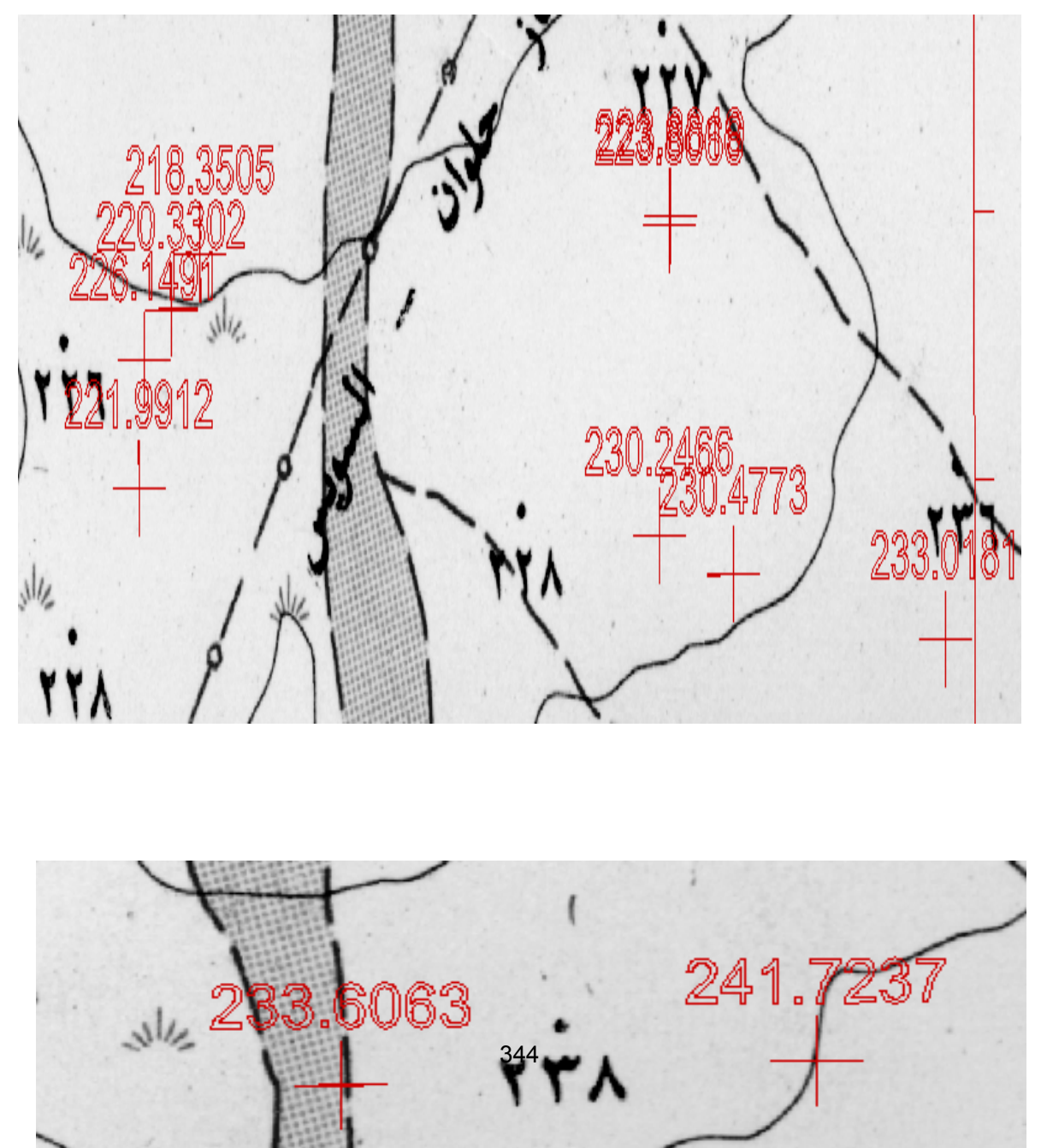
Fig. 3. Overlay of both Digital and Analytical Topographic Maps

Table 1. Specifications of the Scanned Photos

\begin{tabular}{|c|c|c|c|}
\hline \multirow{2}{*}{ Item } & \multicolumn{3}{|c|}{ The Used Scanning Resolution (dpi) } \\
\cline { 2 - 4 } & $\mathbf{2 1 1 7}$ & $\mathbf{1 5 8 8}$ & $\mathbf{1 0 1 6}$ \\
\hline Image Coverage $(\boldsymbol{\mu m})$ & 12 & 16 & 25 \\
\hline $\begin{array}{c}\text { Ground Coverage } \mathbf{( m \mathbf { x }} \\
\mathbf{m})\end{array}$ & $0.18 \times 0.18$ & $0.24 \times 0.24$ & $0.375 \times 0.375$ \\
\hline File Size (MB) & 346.19 & 194.79 & 79.73 \\
\hline
\end{tabular}

Table 2. Statistical Parameters of the Resulted Check Points Discrepancies Corresponding to Each Scanning Resolution

\begin{tabular}{|c|c|c|c|c|}
\hline \multirow{2}{*}{\multicolumn{2}{|c|}{ Ststistical Paramters }} & \multicolumn{3}{|c|}{ Different Used Scanning Resolution (dpi) } \\
\hline & & 2117 & 1588 & 1016 \\
\hline \multirow{2}{*}{$\bar{\Delta}\left\{\begin{array}{c}\mathrm{X}- \\
\text { Direction }\end{array}\right.$} & Max. & 1.2023 & 0.9150 & 2.0409 \\
\hline & Min. & -1.3601 & -0.6201 & -1.4964 \\
\hline
\end{tabular}




\begin{tabular}{|c|c|c|c|c||}
\hline \multirow{4}{*}{} & Range & 2.5624 & 1.5351 & 3.5373 \\
\cline { 2 - 5 } & RMS & 0.8626 & 0.5035 & 1.2406 \\
\hline \multirow{4}{*}{$\begin{array}{c}\text { Y- } \\
\text { Direction }\end{array}$} & Max. & 1.4286 & 1.0035 & 2.8128 \\
\cline { 2 - 5 } & Min. & -1.1965 & -0.9229 & -0.698 \\
\cline { 2 - 5 } & Range & 2.6251 & 1.9264 & 3.5113 \\
\cline { 2 - 5 } & RMS & 0.8868 & 0.5927 & 1.0532 \\
\hline \multirow{4}{*}{$\begin{array}{c}\text { Z- } \\
\text { Direction }\end{array}$} & Max. & 1.8335 & 2.7086 & 5.1570 \\
\cline { 2 - 5 } & Min. & -1.9570 & -2.7008 & -1.4991 \\
\cline { 2 - 5 } & Range & 3.7905 & 5.4094 & 6.6561 \\
\cline { 2 - 5 } & RMS & 1.2629 & 1.7945 & 1.9643 \\
\hline \multirow{3}{*}{$\begin{array}{c}\text { Spatial } \\
\text { position }\end{array}$} & Max. & 2.6168 & 2.8516 & 5.5540 \\
\cline { 2 - 5 } & Min. & 0.7075 & 0.8423 & 0.2464 \\
\cline { 2 - 5 } & Range & 1.9111 & 2.0093 & 5.3076 \\
\cline { 2 - 5 } & RMS & 1.7697 & 1.9558 & 2.5508 \\
\hline
\end{tabular}

Table 3. The Assessment of the Output Exterior Orientation Parameters of the Block Corresponding to Each Scanning Resolution

\begin{tabular}{|c|c|c|c|c|c|c|}
\hline \multirow{3}{*}{$\begin{array}{c}\text { Exterior } \\
\text { Orientation } \\
\text { Parameter }\end{array}$} & \multicolumn{6}{|c|}{ Different Used Scanning Resolution (dpi) } \\
\hline & \multicolumn{2}{|c|}{2117} & \multicolumn{2}{|c|}{1588} & \multicolumn{2}{|c|}{1016} \\
\hline & Residual & Accuracy & Residual & Accuracy & Residual & Accuracy \\
\hline$\overline{X_{L}(\mathrm{~m})}$ & 0.0003 & 3.5596 & 1.0000 & 4.4547 & 1.1014 & 7.8450 \\
\hline$Y_{L}(m)$ & 0.0001 & 3.4363 & 0.8955 & 4.2781 & 6.6906 & 7.8863 \\
\hline $\mathrm{Z}_{\mathrm{L}}(\mathrm{m})$ & 0.0001 & 3.1898 & 0.9551 & 3.9786 & 3.2032 & 6.8568 \\
\hline$\omega$ (deg.) & 0.0001 & 0.0640 & 0.0306 & 0.0793 & 0.1365 & 0.1512 \\
\hline$\varphi$ (deg.) & 0.0001 & 0.0682 & 0.0187 & 0.0855 & 0.0159 & 0.1517 \\
\hline K (deg.) & 0.0001 & 0.0469 & 0.0064 & 0.0584 & 0.0157 & 0.1009 \\
\hline
\end{tabular}

Table 4. Statistical Parameters of the Selected Tie Points Corresponding to Each Scanning Resolution

\begin{tabular}{|c|c|c|c|c|c|c|c|c|c|}
\hline Statistical & \multicolumn{8}{c|}{ Different Used Scanning Resolution (dpi) } \\
\cline { 2 - 10 } $\begin{array}{c}\text { Parameter } \\
(\mathrm{m})\end{array}$ & $\mathrm{X}$ & $\mathrm{Y}$ & $\mathrm{Z}$ & $\mathrm{X}$ & $\mathrm{Y}$ & $\mathrm{Z}$ & $\mathrm{X}$ & $\mathrm{Y}$ & $\mathrm{Z}$ \\
\hline & $\mathrm{X}$ & - & -117 & -128 & 0.007 & 0.030 & 0.228 \\
\hline Mean & - & - & 0.119 & - & - & 0.128 & & & \\
& 0.004 & 0.007 & & 0.010 & 0.015 & & & & \\
\hline Min. & - & - & 0.005 & - & - & 0.009 & - & - & 0.016 \\
\hline
\end{tabular}




\begin{tabular}{|c|c|c|c|c|c|c|c|c|c|}
\hline & 1.755 & 2.701 & & 2.176 & 1.975 & & 3.853 & 6.486 & \\
\hline Max. & 1.096 & 2.709 & 2.852 & 1.690 & 1.834 & 2.747 & 2.855 & 5.157 & 6.637 \\
\hline St.Dev. & 0.202 & 0.396 & 0.456 & 0.304 & 0.321 & 0.495 & 0.485 & 0.750 & 0.954 \\
\hline $\begin{array}{c}\text { Unit- } \\
\text { Weight } \\
\text { (mm) }\end{array}$ & \multicolumn{3}{|c|}{0.0175} & \multicolumn{3}{|c|}{0.0289} & \multicolumn{3}{|c|}{0.0769} \\
\hline $\begin{array}{l}\text { No. of } \\
\text { Points }\end{array}$ & \multicolumn{3}{|c|}{201} & \multicolumn{3}{|c|}{182} & \multicolumn{3}{|c|}{175} \\
\hline
\end{tabular}

